\title{
Anthropometric parameters and liver histology influence lipid metabolic changes in HCV chronic hepatitis on direct-acting antiviral treatment
}

\author{
Domenico Iossa ${ }^{1}$, Martina Vitrone ${ }^{1}$, Massimo Gagliardi ${ }^{1}$, Erasmo Falco ${ }^{2}$, Enrico Ragone ${ }^{2}$, \\ Rosa Zampino ${ }^{1,2}$, Emanuele Durante-Mangoni ${ }^{1,2}$ \\ ${ }^{1}$ Internal Medicine, University of Campania “L. Vanvitelli”, Naples, Italy; ${ }^{2}$ Units of Infectious \& Transplant Medicine AORN dei Colli, Monaldi \\ Hospital, Naples, Italy \\ Contributions: (I) Conception and design: D Iossa, R Zampino, E Durante-Mangoni; (II) Administrative support: None; (III) Provision of study \\ materials or patients: M Vitrone, M Gagliardi; (IV) Collection and assembly of data: M Vitrone, M Gagliardi, E Falco, E Ragone; (V) Data analysis \\ and interpretation: D Iossa, M Gagliardi; (VI) Manuscript writing: All authors; (VII) Final approval of manuscript: All authors. \\ Correspondence to: Emanuele Durante-Mangoni, MD PhD. Department of Precision Medicine, University of Campania, Monaldi Hospital, Ettore \\ Ruggieri Square, Naples 80131, Italy. Email: emanuele.durante@unicampania.it.
}

\begin{abstract}
Background: Hepatitis $\mathrm{C}$ virus $(\mathrm{HCV})$ infection affects lipid metabolism. We investigated the impact of direct-acting antiviral (DAA) treatment on lipid metabolism in chronic hepatitis $\mathrm{C}$ (CHC), with a focus on the effects of anthropometric parameters and liver histology. We also analyzed the dynamics of metabolic indexes used to estimate cardiovascular risk.

Methods: In 49 patients with CHC treated with DAAs, lipid metabolic changes, anthropometric parameters, liver histology and cardiovascular risk indexes, including triglyceride to HDL ratio (Tr/HDL), fatty liver index (FLI) and visceral adiposity index (VAI) were evaluated at baseline (BL), end of treatment (EOT) and 12 [sustained virological response (SVR) 12] and 24 (SVR24) weeks after EOT.

Results: SVR occurred in 96\% of cases. Total and LDL cholesterol and ApoB levels increased significantly between $\mathrm{BL}$ and EOT $(\mathrm{P}<0.001,<0.001$ and 0.05 , respectively) and remained stable thereafter. Total and LDL cholesterol significantly increased only in patients with higher $\mathrm{BL}$ waist circumference $(\mathrm{P}<0.01$ and 0.009), fibrosis $(\mathrm{P}=0.002$ and 0.005$)$ and steatosis $(\mathrm{P}=0.043$ and 0.033 , respectively). HDL cholesterol significantly rose at SVR24. However, cardiovascular risk indexes (Tr/HDL ratio, FLI and VAI) did not significantly change during DAA treatment and follow up.

Conclusions: Patients with HCV eradication after DAA treatment develop a pro-atherogenic lipid pattern, which varies according to anthropometric parameters and liver histology. However, no increase of cardiovascular risk indexes occurs in the short-term. Total and LDL cholesterol should be monitored longterm in $\mathrm{CHC}$ patients cured from infection.
\end{abstract}

Keywords: Body mass index (BMI); direct-acting antiviral treatment (DAA treatment); hepatitis C virus (HCV); lipid; waist circumference

Submitted Jan 13, 2020. Accepted for publication Jul 31, 2020.

doi: 10.21037/atm-20-669

View this article at: http://dx.doi.org/10.21037/atm-20-669

\section{Introduction}

Hepatitis $\mathrm{C}$ virus (HCV) is a major cause of liver disease worldwide. Chronic infection occurs due to the high propensity of the virus to chronically persist in the host, leading to cirrhosis, hepatocellular cancer, diabetes mellitus and atherosclerosis (1-3).

$\mathrm{HCV}$ is present in the extracellular compartment in close interaction with lipoproteins, forming the so called 
'lipoviroparticles'. This allows HCV to escape from antibody neutralization, bind to the low-density lipoprotein receptor and enter hepatocytes (4).

Important pathophysiological steps of $\mathrm{HCV}$ infection are also related to virion interaction with lipids within hepatocytes (5). HCV particle maturation requires binding to lipid rafts and exploits hepatocyte lipoprotein secretion mechanisms $(6,7)$, effectively impairing very lowdensity lipoprotein (VLDL) production. This translates into hypolipidemia as well as lipid accumulation within hepatocytes, causing liver steatosis (8).

Likely due to these interactions, $\mathrm{HCV}$ infection is generally associated with a reduced serum level of total cholesterol, LDL cholesterol and ApoB (9). Viral clearance with early Interferon-based regimens was shown to translate into resolution of this form of hypobetalipoproteinemia, but this effect could be attenuated by the nutritional changes and weight loss commonly occurring on interferon treatment.

The availability of direct-acting antivirals (DAA) for $\mathrm{HCV}$ treatment made possible to better assess the effects of antiviral therapy on host lipid metabolism $(10,11)$. HCV RNA clearance associated with increased cholesterol levels, particularly LDL, without major effects on triglycerides $(10,12,13)$, confirming the direct role of HCV on ApoBrelated lipid metabolic changes. The resulting lipid pattern, however, turns to a more atherogenic one, thereby possibly increasing cardiovascular risk long term. Accordingly, lipid pattern should be evaluated and monitored in all $\mathrm{HCV}$ patients who clear the virus on DAAs.

It is well known that body weight and body composition significantly affect mortality and cardiovascular risk, even in the absence of metabolic syndrome $(14,15)$. Furthermore, a worse metabolic pattern is often correlated to changes in body mass index (BMI) and waist circumference $(16,17)$, whilst weight loss usually produces beneficial lipid profile changes in the medium term (18). This is an important issue in DAA-treated $\mathrm{HCV}$ patients as viral clearance is often associated with favorable behavioral changes and a psychological wellbeing that may increase food intake. Notwithstanding, the role of anthropometric parameters in relation to changes of lipid profile during DAA treatment has not been fully assessed $(10,12,13)$.

In the present study, we evaluated the impact of DAA treatment on lipid metabolism in patients with chronic hepatitis $\mathrm{C}(\mathrm{CHC})$ at the end of therapy and during followup. In this context, we specifically analyzed the effect of anthropometric parameters on such metabolic changes and the dynamics of metabolic indexes used to measure cardiovascular risk.

We present the following article in accordance with the STROBE reporting checklist (available at http://dx.doi. org/10.21037/atm-20-669).

\section{Methods}

\section{Study cohort}

This was a retrospective, observational study on 49 consecutive patients with CHC cared for at the Unit of Infectious \& Transplant Medicine, University of Campania "L. Vanvitelli", Monaldi Hospital in Naples, Italy. Fortynine patients were enrolled from June 2015 to June 2016. All study procedures were in accordance with international guidelines, standards on human experimentation of the Ethics Committee of the Luigi Vanvitelli University and 1975 Helsinki Declaration (as revised in 2013) and subsequent revisions. The study protocol was approved by our University Ethics Committee (No. 662/17). Informed consent was taken from all the patients.

\section{Clinical and laboratory studies}

Diagnosis of $\mathrm{CHC}$ hinged on serological and virological tests (positive serum anti-HCV antibodies and HCV RNA), liver injury markers and liver ultrasound scan.

Patients underwent complete physical examination and were evaluated at baseline (BL), at the end of treatment (EOT) and 12 and 24 weeks after the EOT. Sustained Virological Response was obtained when HCVRNA was negative at 12 (SVR 12) and 24 (SVR24) weeks after the EOT. AST and ALT are considered as markers of liver injury. The BMI (as weight in kilograms/height2 in meters) and waist circumference (WC, normal $<102 \mathrm{~cm}$ in men and $<88 \mathrm{~cm}$ in women) (19) were measured at the 4 study time points.

Liver function tests, HCV-RNA, glycemia, hemoglobin $\mathrm{A} 1 \mathrm{c}$ and the lipid profile (total cholesterol, HDL cholesterol, LDL cholesterol, triglycerides, ApoA1 and ApoB100) were measured at all study time points. VLDL cholesterol was calculated by the Friedewald formula (i.e., serum triglycerides/5) (20). Attention was given to a prior history of diabetes and the concurrent therapies that could interfere with lipid levels.

HCV RNA, HCV genotype, HBV and HIV infection markers were also analyzed by our Hospital central 
laboratory using standard methods.

Liver histology, with evaluation of histological activity index (HAI), fibrosis and steatosis, was assessed in 15 of 49 patients $(30.6 \%)$ by standard methods $(21,22)$; where liver biopsy was not done for contraindications or patient refusal, fibrosis was non-invasively assessed by transient elastography (TE, Fibro Scan ${ }^{\circledR}$, EchoSens, Paris, France). Steatosis was evaluated in all patients by the ultrasonographic fatty liver indicator (US-FLI), where a score $>2$ indicated the presence of steatosis $(23,24)$. Furthermore, the Fatty Liver Index (FLI), based on triglycerides, BMI, WC and $\gamma$-glutamyltranspeptidase (GGT) (25) was measured at the 4 study time points. A FLI $<30$ ruled out and one $\geq 60$ ruled in hepatic steatosis as detected by ultrasonography.

To estimate cardiovascular risk related to metabolic alterations and/or visceral obesity at BL and during followup, the triglycerides-to-HDL ratio (Tr/HDL) (26-28) and the visceral adiposity index (VAI) (29) were calculated. High values of $\mathrm{Tr} / \mathrm{HDL}$ are predictive of coronary heart/ cardiovascular disease and related mortality. Values $\leq 2$ are considered normal. VAI is a sex-specific index, based on WC, BMI, triglycerides, and HDL cholesterol that indirectly expresses visceral fat function. In healthy, non-obese subjects, with normal adipose distribution and normal TG and HDL levels, VAI should be equal to 1 (29). The presence of Metabolic Syndrome (MetS) was defined according to the revised National Cholesterol Education Program Adult Treatment Panel (NCEP-ATP) III criteria (19).

\section{Treatment}

Patients started treatment according with the current guidelines (29) and using drugs available in Italy at the time the study was performed. Five patients received sofosbuvirribavirin (400/1,200 mg daily), 8 sofosbuvir-daclatasvir (400/60 mg daily), 18 sofosbuvir-ledipasvir (400/90 mg daily) and 17 ombitasvir/paritaprevir/ritonavir-dasabuvir (25/150/100/500 mg/daily). Ribavirin (1,200 mg) was added to the DAA regimen in 25 additional patients, as per current recommendations (30) and generally for 3 months.

\section{Statistical analysis}

Categorical variables were expressed as numbers and percentages. Continuous variables were expressed as median and range or interquartile range (IQR). The level of significance was fixed at $5 \%$ and only two-tailed, nonparametric tests were used. Categorical variables were compared using Fisher's exact test, while continuous variables were compared using the Kruskal-Wallis test for different subgroups. An analysis of variance for repeated measures was performed using the Bonferroni post-hoc test. All the analyses were conducted using SPSS v.20.0 software.

\section{Results}

\section{Baseline patient characteristics}

Forty-nine patients were studied; all were naive to treatment with DAAs, while 13 of them had been previously treated with pegylated-interferon and ribavirin, without virological response.

Baseline characteristics of the study group are shown in Table 1. Patients' median age was 66 years, with a slight predominance of women. On average, patients were overweight at baseline (median BMI 26.7) and the median $W C$ was $>100 \mathrm{~cm}$.

HCV genotype 1 was prevalent $(75.5 \%)$ and no patient had HBV or HIV co-infection.

Fibrosis stage was mostly elevated (median fibrosis score $3)$ while steatosis was mild (median score 2 ). Based on a FLI $\geq 60$, steatosis was present in 24 patients (48.9\%) whilst MetS was noted in 14 patients $(28.5 \%)$ at BL.

At BL, glycemia and hemoglobin A1c were within normal values in most patients (Table 1). Eight patients (16.3\%) had a prior diagnosis of diabetes mellitus. Only 3 subjects $(6 \%)$ were on treatment with a hydroxyl-methylglutaryl-coenzyme A reductase inhibitor.

\section{Outcome of treatment}

All patients showed undetectable HCVRNA at EOT. SVR 12 and SVR 24 were achieved in 47 patients (96\%), with 2 patients experiencing a relapse of infection after ontreatment serum HCV RNA clearance.

Overall, regimens were well tolerated, without treatmentemergent ALT, GGT, bilirubin, glycemia or prothrombin time alterations; no signs of hepatic decompensation or renal impairment were observed.

\section{Lipid changes during $\mathrm{DA} A$ treatment}

Significant differences were observed for total cholesterol, LDL cholesterol and glycated hemoglobin at the four study time points $(\mathrm{P}=0.005,0.008$ and $<0.001$, respectively; Table 1). The post-hoc tests showed that total cholesterol 
Table 1 The baseline and follow-up clinical characteristics of study patients

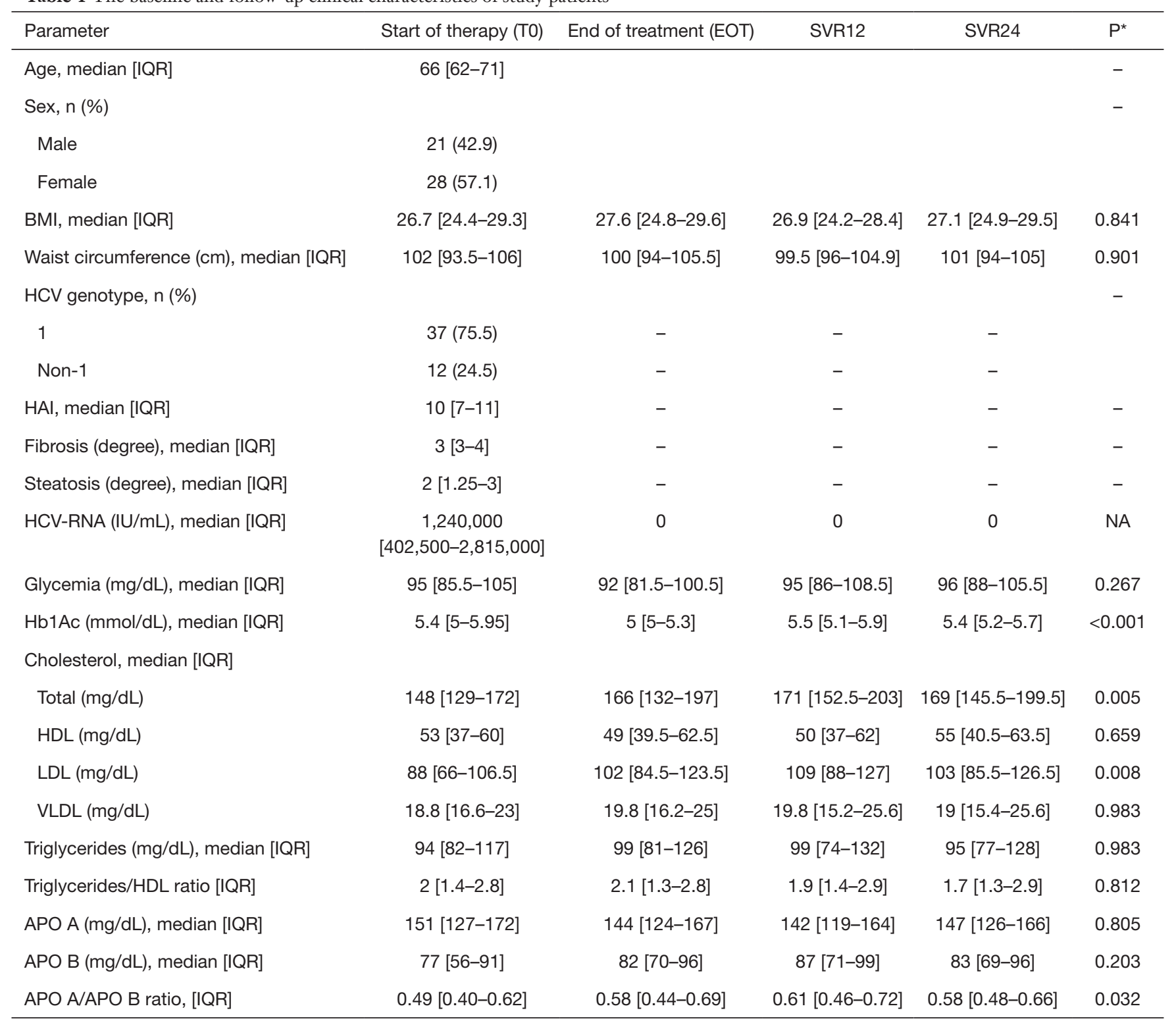

*, samples Kruskal-Wallis test; IQR, interquartile range; BMI, body mass index; HAI, histological activity index; Hb1Ac, glycated hemoglobin;

levels increased significantly between $\mathrm{BL}$ and the EOT $(\mathrm{P}<0.001)$. After the EOT, no further significant changes were observed up to SVR24. In summary, total cholesterol increased during DAA treatment from baseline to EOT, subsequently reaching a plateau (Figure 1).

LDL cholesterol levels paralleled those of total cholesterol, with a significant increase from BL to EOT and a subsequent plateau (Figure 1).

In contrast, HDL cholesterol changes were less pronounced, with an overall trend towards reduction from BL to SVR12 and a subsequent significant rise at SVR24. Overall, HDL levels were significantly higher at SVR24 compared to BL $(\mathrm{P}=0.018)$ and SVR12 $(\mathrm{P}=0.046)$. VLDL cholesterol did not change throughout the observation period (Figure 1).

No significant differences in triglyceride and Apo-A levels were observed at any time-point (Figure 1), while Apo-B levels significantly increased from BL to EOT 

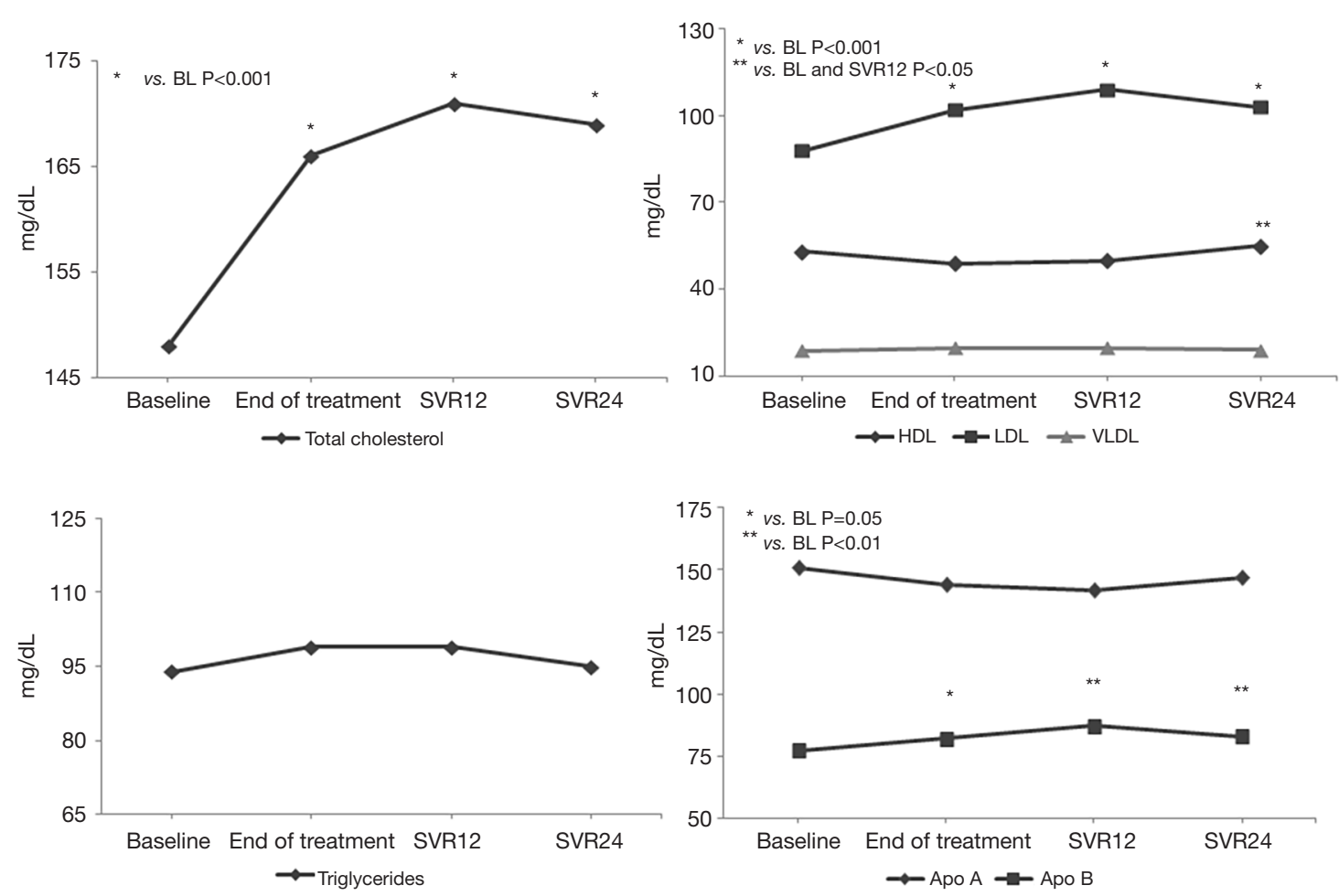

Figure 1 Lipid profile changes on DAA treatment. Statistical comparisons were performed with analysis of variance with Bonferroni's correction. P values are shown on the graphs. * and ** denote the relevant time points. BL, baseline; SVR, sustained virological response (at weeks 12 or 24).

$(\mathrm{P}=0.05)$, SVR 12 and SVR24 $(\mathrm{P}<0.01$, Figure 1$)$. In line with these findings, ApoB/ApoA ratio also significantly increased during the observation period $(\mathrm{P}=0.032$; Table 1).

Interestingly, when we specifically evaluated the 2 patients who did not achieve SVR, we did not observe any significant variation of their lipid profile (data not shown).

No significant differences were observed between patients treated with sofosbuvir and those treated with nonsofosbuvir based regimens (data not shown). Likewise, ribavirin did not influence metabolic changes (data not shown).

\section{Relationship between lipid changes, liver fibrosis and liver steatosis}

We assessed whether lipid changes on DAA treatment occurred differently according to BL liver histology. Both total and LDL cholesterol increased significantly (Table 2) only in patients with BL liver cirrhosis and in those with steatosis, i.e., showing an US-FLI score $>2$. In contrast, there was no difference in the dynamics of HDL cholesterol and triglycerides according to liver histopathological stage (Table 2). As total and LDL cholesterol increase could pair with an increased release of lipids from HCV clearing hepatocytes, we assessed whether lipid changes were paralleled by a reduction in US-FLI. However, US-FLI did not significantly change during DAA treatment and follow up. Furthermore, no correlation emerged between USFLI and LDL cholesterol levels at any time point (data not shown).

\section{Relationship between lipid changes and anthropometric parameters}

We then evaluated whether anthropometric parameters affected lipid metabolic changes during DAA. Importantly, no significant variation in BMI and WC was observed during treatment and until week 24 follow-up (Table 1). Yet, anthropometric parameters appeared to influence lipid changes. As shown in Figure 2, LDL cholesterol significantly increased only in patients with higher BMI and WC at BL (Figure 2). Furthermore, total cholesterol increased only in 
Table 2 Lipid metabolic changes according to the presence or absence of liver cirrhosis and liver steatosis

\begin{tabular}{|c|c|c|c|c|c|c|}
\hline Serum lipid fractions & Clinical status & Start of therapy (T0) & End of treatment EOT) & SVR12 & SVR24 & $P^{*}$ \\
\hline \multirow{2}{*}{ LDL cholesterol } & Yes $(n=31)$ & $86[39-140]$ & 95 [47-145] & 99 [49-157] & $98[40-154]$ & 0.005 \\
\hline & No $(n=18)$ & 103 [58-174] & $113.5[72-199]$ & $118.5[74-187]$ & $107.5[72-216]$ & 0.579 \\
\hline \multirow[t]{2}{*}{ HDL cholesterol } & CIRRHOSIS & & & & & \\
\hline & No $(n=18)$ & $52.5[31-84]$ & $52[30-81]$ & 42 [34-69] & 55 [36-79] & 0.954 \\
\hline \multirow[t]{3}{*}{ Total cholesterol } & CIRRHOSIS & & & & & \\
\hline & Yes $(n=31)$ & 138 [72-217] & 160 [88-225] & 168 [89-224] & 167 [86-252] & 0.002 \\
\hline & No $(n=18)$ & 167.5 [110-258] & 180.5 [112-288] & 179 [123-271] & $172.5[110-312]$ & 0.562 \\
\hline Triglycerides & No $(n=18)$ & $96[51-161]$ & $95.5[74-136]$ & 95 [47-143] & 94 [40-172] & 0.578 \\
\hline \multirow[t]{3}{*}{ LDL cholesterol } & STEATOSIS & & & & & \\
\hline & Yes $(n=18)$ & $86[39-174]$ & 95 [49-199] & 114 [52-187] & 98 [64-216] & 0.043 \\
\hline & No $(n=29)$ & $91[39-154]$ & 107 [47-162] & 105 [49-169] & 104 [40-162] & 0.117 \\
\hline \multirow[t]{3}{*}{ HDL cholesterol } & STEATOSIS & & & & & \\
\hline & Yes $(n=18)$ & 39.5 [15-84] & 45.5 [30-81] & 40 [29-77] & $47.5[31-79]$ & 0.672 \\
\hline & No $(n=29)$ & 55 [18-89] & 50 [25-101] & 54 [24-91] & 59 [25-92] & 0.798 \\
\hline Total cholesterol & STEATOSIS & & & & & \\
\hline
\end{tabular}

All values shown are median [range]. ${ }^{*}$, independent samples Kruskal-Wallis test.

patients with a higher BL WC (Figure 2).

\section{Changes in lipid-related cardiovascular risk markers during $D A A$ treatment}

Tr/HDL ratio, FLI and VAI (Table 1, Figure 3, respectively) did not significantly change during DAA treatment and follow up, indicating no significant increase of estimated cardiovascular risk.

Of note, total and LDL cholesterol changes during DAA therapy did not show any association with BL FLI $(<60$ or $\geq 60)$ or VAI $(\leq 1.49$ or $>1.49$, the median value for the studied group) (Table 3). Similarly, the presence of MetS at
BL did not influence lipid changes (Table 3). However, total cholesterol increased significantly only in patients with a BL Tr/HDL ratio $>2(\mathrm{P}=0.025)$.

\section{Discussion}

In this study, we analyzed the dynamics of circulating lipid profile in patients treated with DAA for CHC. In line with previous reports $(10,12,13,31)$, total and LDL cholesterol levels increased in parallel to HCV RNA clearance. These changes persisted during the subsequent follow-up, indicating that a different, more 'atherogenic' lipid pattern establishes after CHC cure. This effect was independent 
Table 3 Changes in total and LDL cholesterol during DAA therapy in patients grouped according to cardiovascular risk markers

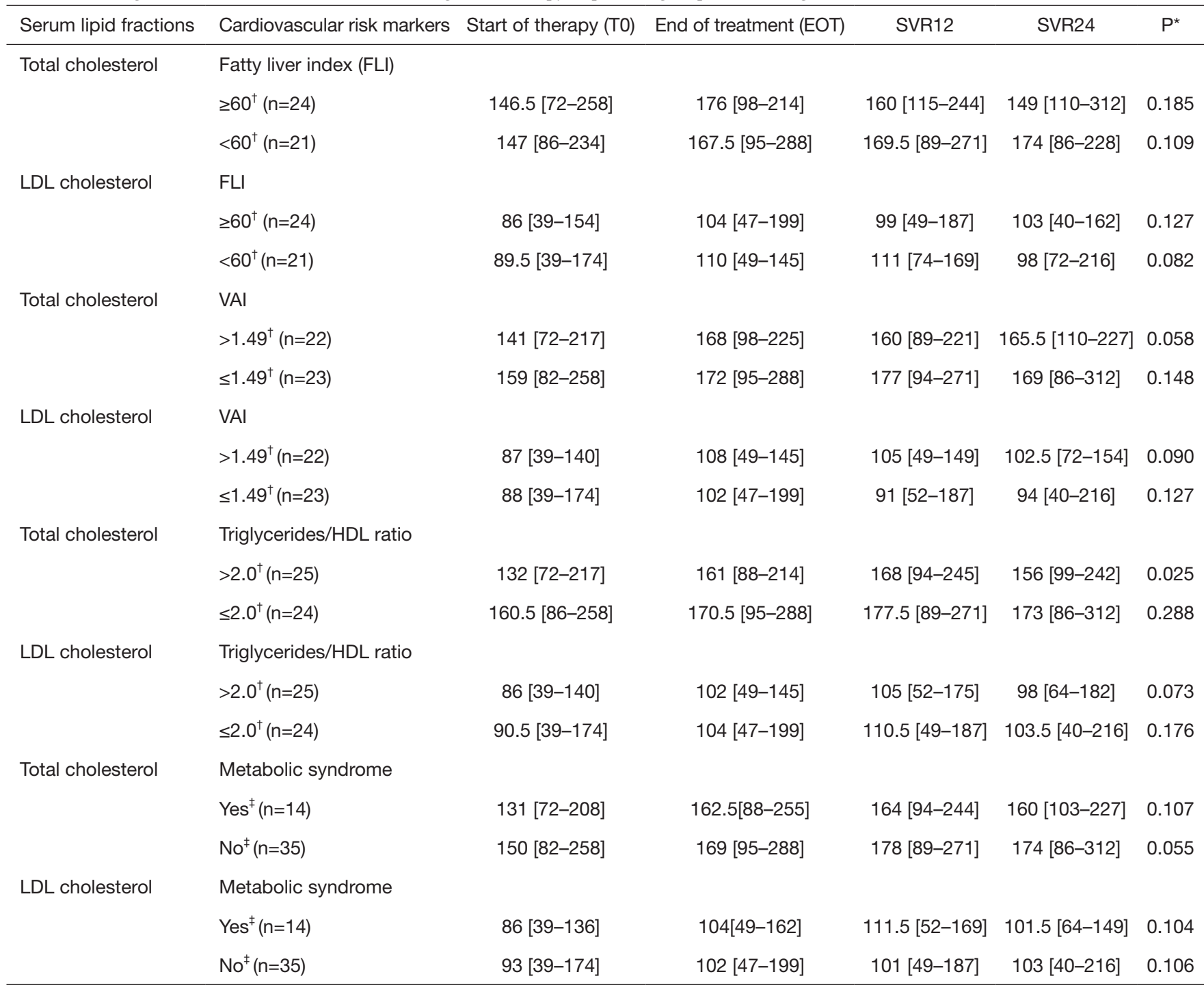

All values shown are median [range]. ${ }^{*}$, independent samples Kruskal-Wallis test; ${ }^{\dagger}$, median value at baseline; ${ }^{\ddagger}$, presence, or absence at baseline. FLI, fatty liver index; VAI, visceral adiposity index.

of the treatment regimen and essentially resembled what had been previously observed in interferon-treated patients $(10,32,33)$.

Novel data emerge from our analyses. First, lipid changes are influenced by liver histological stage and body composition. Although BMI and waist circumference did not change during the observation period, total and LDL cholesterol increase was significant in patients who were overweight/obese and with a higher grade of fibrosis and steatosis at DAA treatment start. This is particularly relevant in terms of $\mathrm{CHC}$ pathophysiology as hepatocyte lipid accumulation is considered a major cause of liver injury and a driver of liver disease progression (34). Hence, the pronounced changes in lipid levels after HCV clearance in those with worse liver disease indirectly confirm HCV is the major player of intracellular lipid trafficking impairment and that this mechanism possesses a true pathological significance. Indeed, the 2 patients who did not achieve SVR did not show any modification of serum lipid levels.

It is interesting to note that most of our patients had $\mathrm{CHC}$ due to $\mathrm{HCV}$ genotypes 1 and 2. At variance with infection due to genotype 3 , liver steatosis is more strictly 

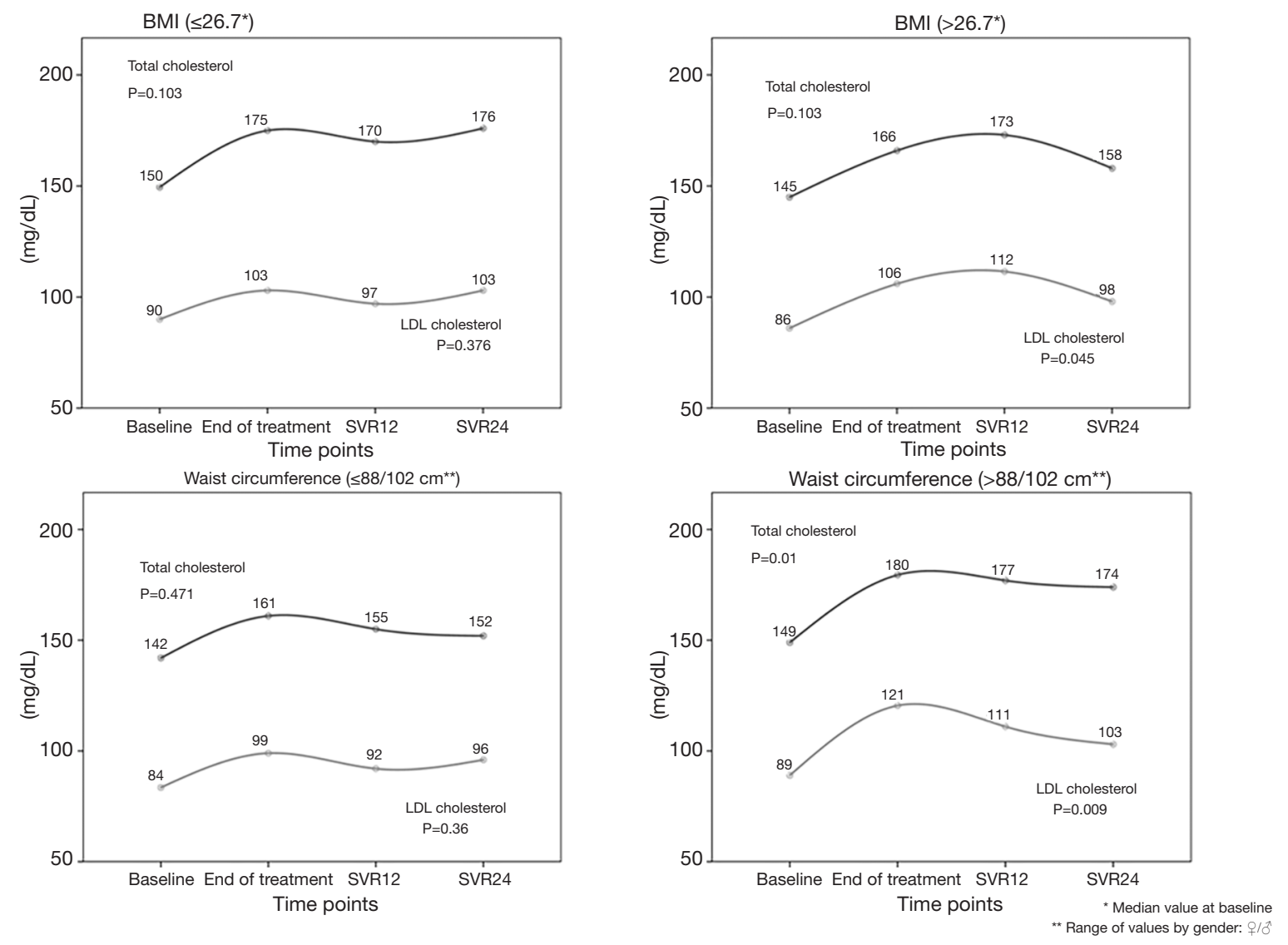

Figure 2 Lipid profile changes according to anthropometric parameters at different time points during DAA treatment. Statistical comparisons were performed with Kruskal-Wallis test. P values are shown on the graphs. SVR, sustained virological response (at weeks 12 or 24).

associated with body composition in CHC genotypes 1 and 2 (35). This is again in agreement with our findings, showing lipid changes during DAA are more pronounced in overweight/obese and viscerally obese patients. This further suggests that in CHC, patient weight and body fat should be kept under control to maintain lipid homeostasis.

Our study has limitations. A relatively small number of subjects has been included and this could affect the statistical significance of differences observed. Patients have been treated between 2015 and 2016, when DAAs were prioritized for advanced fibrosis or cirrhosis patients, introducing a selection bias. However, other studies showed similar results in populations with a wide range of liver disease stages $(10,12)$. Mostly HCV genotype 1 patients were included, thus precluding the generalization of our findings to other HCV genotypes, especially genotype 3.

It is known that HCV is involved in atherosclerosis development by a direct and pro-inflammatory effect (35-37). Also, HCV clearance was shown to be associated with a reduction of atherosclerosis lesions in the short term (38). These data are apparently in contrast with the establishment of a more atherogenic lipid pattern emerging from this and prior studies $(10,12,13)$. Therefore, DAA treatmentemergent changes in lipid profile could still play a role in maintaining some residual atherosclerotic or cardiovascular risk in the short or medium term. Although a definitive answer to this issue would only come from larger prospective studies, we aimed to assess whether surrogate markers of cardiovascular risk worsened after DAA treatment. In our study, scores predicting cardiovascular risk, such as VAI, FLI and Tr/HDL, did not show significant modifications up to week 24 after HCVRNA clearance. However, total cholesterol significantly increased only among those with a higher Tr/HDL ratio. Overall, the cumulative analysis of surrogate markers of cardiovascular 

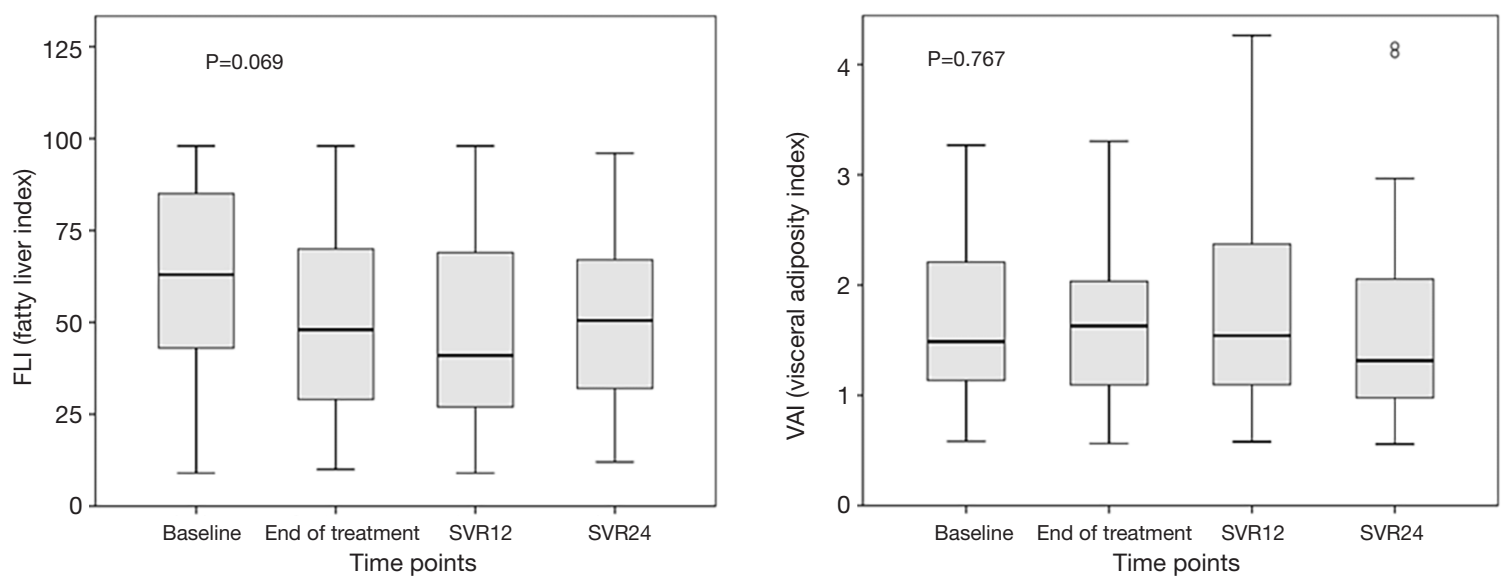

Figure 3 Changes in lipid-related cardiovascular risk markers during DAA treatment. Statistical comparisons were assessed with KruskalWallis test. P values are shown on the graphs. SVR, sustained virological response (at weeks 12 or 24).

risk showed that, at least in the short term, cardiovascular risk after DAA treatment does not change despite of the increase in total and LDL cholesterol levels. Time of observation is probably too short to detect important modifications of these parameters, but it is probable that overweight and particular visceral obesity play a role in the lipid pattern changes during and soon after DAA treatment.

In the next future, patients with SVR to DAA may need specific cardiovascular risk stratification (13) and easily validated predictors, as VAI and Tr/HDL, should enter clinical practice in those with long term SVR. Early detection of an increased cardiovascular risk would favor indication to specific treatments, as statins, in a population previously considered at low risk for hypercholesterolemia and lacking a stringent need for lipid treatment.

\section{Conclusions}

In conclusion, patients with HCV eradication after DAA treatment develop a more atherogenic lipid pattern, that is influenced by anthropometric parameters and liver histology. This, however, does not increase the cardiovascular risk in the short term. Total and LDL cholesterol should be monitored during the long-term follow-up after DAA treatment to implement the correct behavioral and pharmacological strategies.

\section{Acknowledgments}

The authors thanks Ms Lidia Mazza e Mr Roberto Loreto for their nursing assistance.
Funding: None.

\section{Footnote}

Reporting Checklist: The authors have completed the STROBE reporting checklist. Available at http://dx.doi. org/10.21037/atm-20-669

Data Sharing Statement: Available at http://dx.doi. org/10.21037/atm-20-669

Conflicts of Interest: All authors have completed the ICMJE uniform disclosure form (available at http://dx.doi. org/10.21037/atm-20-669). EDM report that received speaker's honoraria from Abbvie and Rosa Zampino received a research grant from Gilead. The authors have no other conflicts of interest to declare.

Ethical Statement: The authors are accountable for all aspects of the work in ensuring that questions related to the accuracy or integrity of any part of the work are appropriately investigated and resolved. All study procedures were in accordance with international guidelines, standards on human experimentation of the Ethics Committee of the Luigi Vanvitelli University and 1975 Helsinki Declaration (as revised in 2013) and subsequent revisions. The study protocol was approved by our University Ethics Committee (No. 662/17). Informed consent was taken from all the patients.

Open Access Statement: This is an Open Access article distributed in accordance with the Creative Commons 
Attribution-NonCommercial-NoDerivs 4.0 International License (CC BY-NC-ND 4.0), which permits the noncommercial replication and distribution of the article with the strict proviso that no changes or edits are made and the original work is properly cited (including links to both the formal publication through the relevant DOI and the license). See: https://creativecommons.org/licenses/by-nc-nd/4.0/.

\section{References}

1. Petruzziello A, Marigliano S, Loquercio G, et al. Hepatitis $\mathrm{C}$ virus (HCV) genotypes distribution: an epidemiological up-date in Europe. Infectious Agents Cancer 2016;11:53.

2. Seto WK, Lai CL, Fung J, et al. Natural history of chronic hepatitis C: genotype 1 versus genotype 6. J Hepatol 2010;53:444-48.

3. Umumararungu E, Ntaganda F, Kagira J, et al. Prevalence of Hepatitis C Virus Infection and Its Risk Factors among Patients Attending Rwanda Military Hospital, Rwanda. Biomed Res Int 2017;2017:5841272.

4. Albecka A, Belouzard S, Op de Beeck A, et al. Role of lowdensity lipoprotein receptor in the hepatitis $\mathrm{C}$ virus life cycle. Hepatology 2012 55:998-1007.

5. Scheel TK, Rice CM. Understanding the hepatitis $C$ virus life cycle paves the way for highly effective therapies. Nat Med 2013;19:837-49.

6. Gastaminza P, Cheng G, Wieland S, et al. Cellular determinants of hepatitis $\mathrm{C}$ virus assembly, maturation, degradation, and secretion. J Virol 2008;82:2120-29.

7. Huang H, Sun F, Owen DM, et al. Hepatitis C virus production by human hepatocytes dependent on assembly and secretion of very low-density lipoproteins. Proc Natl Acad Sci USA 2007;104:5848-53.

8. Syed GH, Amako Y, Siddiqui A. Hepatitis C virus hijacks host lipid metabolism. Trends Endocrinol Metab 2010;21:33-40.

9. Aizawa Y, Seki N, Nagano T, et al. Chronic hepatitis $\mathrm{C}$ virus infection and lipoprotein metabolism. World $\mathrm{J}$ Gastroenterol 2015 21:10299-313.

10. Mauss S, Berger F, Wehmeyer MH, et al. Effect of antiviral therapy for HCV on lipid levels. Antivir Ther 2017;21:81-8.

11. Rivero-Juarez, A, Camacho A, Brieva T, et al. Differential timing of cholesterol increase during successful HCV therapy: impact of type of drug combination. J Acquir Immune Defic Syndr 2018;78:437-40.

12. Meissner EG, Lee YJ, Osinusi A, et al. Effect of sofosbuvir and ribavirin treatment on peripheral and hepatic lipid metabolism in chronic hepatitis $\mathrm{C}$ virus, genotype 1-infected patients. Hepatology 2015;61:790-801.

13. Gitto S, Cicero AFG, Loggi E, et al. Worsening of Serum Lipid Profile after Direct Acting Antiviral Treatment. Ann Hepatol 2018;17:64-75.

14. Flegal KM, Kit BK, Orpana H, et al. Association of allcause mortality with overweight and obesity using standard body mass index categories: a systematic review and metaanalysis. JAMA 2013;309:71-82.

15. Kramer CK, Zinman B, Retnakaran R. Are metabolically healthy overweight and obesity benign conditions?: A systematic review and meta-analysis. Ann Intern Med 2013;159:758-69.

16. Lin $\mathrm{H}$, Zhang $\mathrm{L}$, Zheng R, et al. The prevalence, metabolic risk and effects of lifestyle intervention for metabolically healthy obesity: a systematic review and meta-analysis: A PRISMA-compliant article. Medicine (Baltimore) 2017;96:e8838.

17. Stelmach-Mardas M, Walkowiak J. Dietary Interventions and Changes in Cardio-Metabolic Parameters in Metabolically Healthy Obese Subjects: A Systematic Review with Meta-Analysis. Nutrients 2016;8:E455.

18. Aucott L, Gray D, Rothnie H, et al. Effects of lifestyle interventions and long-term weight loss on lipid outcomes - a systematic review. Obes Rev 2011;12:412-25.

19. Alberti KG, Zimmet P, Shaw J, IDF Epidemiology Task Force Consensus Group. The metabolic syndrome--a new worldwide definition. Lancet 2005;366:1059-62.

20. Martin SS, Blaha M J, Elshazly MB, et al. Comparison of a Novel Method vs. the Friedewald Equation for Estimating Low-Density Lipoprotein Cholesterol Levels From the Standard Lipid Profile. JAMA, 2013;310:2061-8.

21. Ishak K, Baptista A, Bianchi L, et al. Histological grading and staging of chronic hepatitis. J Hepatol 1995;22:696-9.

22. Kleiner DE, Brunt EM, Van Natta M, et al. Design and validation of a histological scoring system for nonalcoholic fatty liver disease. Hepatology 2005;41:1313-21.

23. Ballestri S, Lonardo A, Romagnoli D, et al. Ultrasonographic fatty liver indicator, a novel score which rules out NASH and is correlated with metabolic parameters in NAFLD. Liver Int 2012;32:1242-52.

24. Ballestri S, Nascimbeni F, Baldelli E, et al. Ultrasonographic fatty liver indicator detects mild steatosis and correlates with metabolic/histological parameters in various liver diseases. Metabolism 2017;72:57-65.

25. Bedogni G, Bellentani S, Miglioli L, et al. The Fatty Liver Index: a simple and accurate predictor of hepatic steatosis in the general population. BMC Gastroenterol 2006;6:33. 
26. Chang TI, Streja E, Soohoo M, et al. Association of Serum Triglyceride to HDL Cholesterol Ratio with All-Cause and Cardiovascular Mortality in Incident Hemodialysis Patients. Clin J Am Soc Nephrol 2017;12:591-602.

27. Vega GL, Barlow CE, Grundy SM, et al. Triglyceride-tohigh-density-lipoprotein-cholesterol ratio is an index of heart disease mortality and of incidence of type 2 diabetes mellitus in men. J Investig Med 2014;62:345-49.

28. Hadaegh F, Khalili D, Ghasemi A, et al. Triglyceride/ HDL-cholesterol ratio is an independent predictor for coronary heart disease in a population of Iranian men. Nutr Metab Cardiovasc Dis 2009;19:401-8.

29. Amato MC, Giordano C, Galia M, et al. Visceral Adiposity Index: a reliable indicator of visceral fat function associated with cardiometabolic risk. Diabetes Care 2010;33:920-2.

30. Pawlotsky JM, Aghemo A, Back D, et al. European Association for Study of Liver. EASL Recommendations on Treatment of Hepatitis C. J Hepatol 2015;63:199-236.

31. Beig J, Orr D, Harrison D, Gane E. Hepatitis C Virus Eradication with New Interferon-Free Treatment Improves Metabolic Profile in Hepatitis C Virus-Related Liver Transplant Recipients. Liver Transpl 2018;24:1031-9.

32. Goossens N, Negro F. Is genotype 3 of the hepatitis C virus the new villain? Hepatology 2014;59:2403-12.

Cite this article as: Iossa D, Vitrone M, Gagliardi M, Falco E, Ragone E, Zampino R, Durante-Mangoni E. Anthropometric parameters and liver histology influence lipid metabolic changes in $\mathrm{HCV}$ chronic hepatitis on direct-acting antiviral treatment. Ann Transl Med 2021;9(1):35. doi: 10.21037/atm-20-669
33. Corey KE, Kane E, Munroe C, et al. Hepatitis C virus infection and its clearance alter circulating lipids: implications for long-term follow-up. Hepatology 2009;50:1030-7.

34. Adinolfi LE, Gambardella M, Andreana A, et al. Steatosis accelerates the progression of liver damage of chronic hepatitis $\mathrm{C}$ patients and correlates with specific $\mathrm{HCV}$ genotype and visceral obesity. Hepatology 2001;33:1358-64.

35. Adinolfi LE, Zampino R, Restivo L, et al. Chronic hepatitis $\mathrm{C}$ virus infection and atherosclerosis: clinical impact and mechanisms. World J Gastroenterol 2014;20:3410-7.

36. Adinolfi LE, Restivo L, Zampino R, et al. Chronic HCV infection is a risk of atherosclerosis. Role of HCV and HCV-related steatosis. Atherosclerosis 2012;221:496-502.

37. Petta S, Torres D, Fazio G, et al. Carotid atherosclerosis and chronic hepatitis $\mathrm{C}$ : a prospective study of risk associations. Hepatology 2012;55:1317-23.

38. Petta S, Adinolfi LE, Fracanzani AL, et al. Hepatitis C virus eradication by direct-acting antiviral agents improves carotid atherosclerosis in patients with severe liver fibrosis. J Hepatol 2018;69:18-24. 